\title{
COMMENTARY
}

\section{TRAIL and Bortezomib: Killing Cancer with Two Stones}

\author{
Muhammad Zahid Qureshi', Mirna Azalea Romero², Rukset Attar ${ }^{3}$, Zeeshan \\ Javed $^{4}$, Ammad Ahmad Farooqi ${ }^{5 *}$
}

\begin{abstract}
Cancer genomics and proteomics have undergone considerable broadening in the past decades and increasingly it is being realized that solid/liquid phase microarrays and high-throughput resequencing have provided platforms to improve our existing knowledge of determinants of cancer development, progression and survival. Loss of apoptosis is a widely and deeply studied process and different approaches are being used to restore apoptosis in resistant cancer phenotype. Modulating the balance between pro-apoptotic and anti-apoptotic proteins is essential to induce apoptosis. It is becoming more understood that pharmacological inhibition of the proteasome might prove to be an effective option in improving TRAIL induced apoptosis in cancer cells. Keeping in view rapidly accumulating evidence of carcinogenesis, metastasis, resistance against wide ranging therapeutics and loss of apoptosis, better knowledge regarding tumor suppressors, oncogenes, pro-apoptotic and anti-apotptic proteins will be helpful in translating the findings from benchtop to bedside.
\end{abstract}

Keywords: Apoptosis - TRAIL - signaling - cancer - bortezomib

Asian Pac J Cancer Prev, 16 (4), 1671-1674

\section{Introduction}

It is becoming sequentially more notable that changes in the transcriptome provides information to cell to compute and buffer rapid shifts in signals being trasduced either from exracellular or intracellular environment, but post-translationally modified proteins provide quicker mechanism for activation or inhibition of signal cascades. Wealth of information suggested that proteasome is a multienzyme complex reportedly involved in regulating degradation of more than $80 \%$ of ubiquitin-tagged cellular proteins. Cell culture, pre-clincal and clinical studies substantiated potent activity of Bortezomib (Velcade, PS-341) as chymotryptic site-selective inhibitor and a potent dipeptide boronic acid in an NCI 60 cell line screen. Bortezomib is an FDA approval drug for multiple myeloma treatment (Bross et al., 2004). Confluence of information indicated that Bortezomib reversibly inhibited 20S proteasome (Ki $0.6 \mathrm{nM}$ ) via targeting threonine residue on the chymotrypsin-like (CT-L) subunit. (Chauhan et al., 2005).

TRAIL induced intracellular signaling has emerged as one amongst the most extensively and deeply studied molecular phenomenon because of its ability to differentially kill cancer cells and while leaving normal cells intact. TRAIL transduces the signals intracellularly through death receptor (DR4 or DR5) that results in receptor trimerization, and recruitment of the adaptor molecule FAS-associated death domain protein (FADD) by interacting with death domains (DD) of DR4 or DR5. FADD recruits procaspase- 8 by means of its death-effector domain (DED) to form a death inducing signaling complex (DISC) consisting of DR, FADD and caspase-8. FLICE-like inhibitory protein (FLIP) exerts inhibitory effects on caspase- 8 activation. Caspase- 8 further activates its downstream effector caspase-3. Mitochondrial pathway is activated by caspase- 8 mediated proteolytic processing of Bid into truncated Bid. BCLXL and B-cell lymphoma 2 (BCL-2) exert inhibitory effects on loss of mitochondrial membrane potential (MMP), however BAX (BCL-2-associated X protein) domains are reported to promote MMP loss. Cytosolically accumulated Second mitochondria-derived activator of caspase (SMAC) and Cytochrome c are consequences of MMP and cytochrome $\mathrm{c}$ interacted with pro-caspase-9 and apoptotic protease-activating factor 1 (APAF1) to form apoptosome. Inhibitor-of-apoptosis proteins (IAPs) mediated inhibition of caspase activation is neutralized by Second mitochondria-derived activator of caspase (SMAC) induced inhibitory effects on IAPs.

Bortezomib induced apoptosis in TRAIL-resistant HPV infected head and neck cancer cells by increasing DR5 expression, cytosolic accumulation of cytochrome c. Moreover, bortezomib functionalized caspase- 8 , caspase- 9 and caspase-3. XIAP depleted cells displayed improved sensitivity to TRAIL induced signaling in HPV infected

${ }^{1}$ Department of Chemistry, GCU Lahore, ${ }^{4}$ Chughtais Lahore Lab, , ${ }^{5}$ Laboratory for Translational Oncology and Personalized Medicine, Rashid Latif Medical College, Lahore, Pakistan, ${ }^{2}$ Laboratorio de Farmacologia, Unidad Academica de Medicina, Universidad Autonoma de Guerrero, Acapulco Guerrero Mexico, ${ }^{3}$ Department of Obstetrics and Gynecology, Yeditepe University Faculty of Medicine, Ankara, Turkey*For correspondence: ammadahmad638@yahoo.com 
cancer cells (Bullenkamp et al., 2014).

\section{P53 Involvement}

Both HCT116 p53-/- and p53+/+ cells had cell surface expression of DcR 1 and DcR2. Surprisingly, DR4 to DR5 ratio was higher in HCT116 p53-/- cells and the level of DcR1 and DcR2was almost twice higher in HCT116 p53-/- cells. Decoy receptors blockade considerably promoted DR5 induced signaling thus empasizing on the fact that DR5 mediated signaling was negatively regulated by decoy receptors. DR5-B is a DR5 targeting variant of TRAIL with notable efficacy in HCT116 p53+/+ but not in p53-/- cells. However it was convincingly revealed that efficacy of DR5-B increased temporally when combined with bortezomib. Mechanistically it was shown that combinatorial treatment temporally shifted intracellular signaling through receptor from DR4 to DR5. Astonishingly, bortezomib mediated effects were higher in HCT116 p53+/+ cells as compared to HCT116 p53-/-. TRAIL and bortezomib induced internalization and degradation of DR4 as evidenced by confocal microscopy experiments with FITC-conjugated antibodies to DR4 (Bychkov et al., 2014).

\section{DISC Formation}

It is intriguing to note that bortezomib remarkably enhanced TRAIL induced intracellular signaling by enhancing DISC formation and exerting inhibitory effects on c-FLIP in primary meningioma cells (Koschny et al., 2014). Treating HeLa cells with Bortezomib revealed that within 2-3 hours post-treatment, both cFLIP and TRAIL-R2 levels increased 3-fold in response to bortezomib. There was a rapid depletion of pools of free cFLIPS,L, upon stimulation with high, saturating concentrations of TRAIL and progressively procaspase- 8 was converted into fully processed, active caspase- 8 time dependently. Increasing the amount of TRAIL receptors promoted rapid caspase-8 activation at high TRAIL doses (Laussmann et al., 2012). Western blot analysis based findings revealed that bortezomib treatment considerably enhanced, cFLIPL cleavage fragment p43, FADD and biologically active caspase- 8 in TRAIL treated cancer cells. Ubiquitination and consequent proteasomal degradation of tBid notably impaired cytosolic accumulation of mitochondrial resident pro-apoptotic proteins. Chemical inhibition of TRAIL induced downstream signaling to effectively moniter formation and stability of $\mathrm{tBid}, \mathrm{T} 98 \mathrm{G}$ cells were treated with TRAIL for 12 hours to induce formation of tBid. Following stimulation, post treated cells were washed and treated with caspase inhibitor to inhibit caspase- 8 mediated proteolytic processing of Bid. In these treatment conditions bortezomib efficiently inhibited tBid degradation and moreover, apoptosis was dramatically reduced in Bid silenced cancer cells (Unterkircher et al., 2011). Combining recombinant TRAIL with TRAIL-R2specific antibodies (conatumumab) effectively enhanced TRAIL induced DISC formation, enabled by synchronous binding of conatumumab and TRAIL to TRAIL-R2 (Tuthill et al., 2014).

\section{Antagonizing Effects of Bortezomib on Biological Effects Induced by different Drugs}

Increasingly it is being realized that Bortezomib protected cancer cells against Taxol-induced apoptosis. More importantly, Bortezomib also rescued cancer cells from Taxol-mediated G2/M arrest. Detailed mechanistic insights indicated that Taxol significantly increased phospho-histone-3 and PLK1 levels, which were reduced considerably by bortezomib. Taxol-induced anti-apoptotic protein MCL-1 degradation was also impaired by Bortezomib (Rapino et al., 2013).

\section{TRAIL Induced Signaling in Bax-deficient Cancer Cells}

In-vitro analysis has provided evidence regarding caspase- 8 mediated proteolytic processing of procaspase-3 only in TRAIL-sensitive HCT116 wt cells. However, caspase- 8 mediated processing of pro-caspase- 3 was not noted in TRAIL-resistant HCT116 Bax-/Bak- cells (Gillissen et al., 2013). Astonishingly, prolonged exposure of TRAIL to Bax-deficient HCT116 cells resulted in loss of their sensitivity to TRAIL predominantly because of downregulated expression of TRAIL receptor. Moreover, it was also noted that TRAIL treatment induced migratory ability in TRAIL treated cancer cells. TRAIL-mediated cleavage of ROCK1 resulted in an increased migration of cancer cells (Somasekharan et al., 2013).

\section{ER Stress}

Endoplasmic Reticulum Stress is a deeply studied molecular mechanism reported to modulate cancer development and cell death. CCAAT/enhancer-binding protein homologous protein (CHOP), a downstream effector of ER) stress induced signaling cascade is reported to stimulate DR5 expression in PS-341 treated NSCLC cells H157, H460 cells and Calu-1 dosedependently. ATF4 silenced cancer cells did not indicate upregulation of CHOP and DR5. Transfecting cells with CHOP and ATF3 encoding lentiviral plasmids in H157 cells indicated that ATF3 was associated with CHOP as evidenced by detection of ATF3 by western blot and immunoprecipitation technique using CHOP antibody. PS-341 treated cancer cells displayed a marked increase in ATF4 expression, that acted as a transcriptional factor, to promote expression of CHOP and ATF3. PS-341 exerted stimulatory effects on DR5 by enhancing p-ERK and p-RSK2 phosphorylation and expectedly, U0126 (MAPERK kinase inhibitor) suppressed DR5 upregulation. It is also intriguing to note that PKC $\delta$ silenced cancer cells had notably reduced phosphorylated levels of ERK1/2 and RSK2 (Xu et al., 2012).

\section{AKT Induced Downstream Signaling}

Malignant pleural mesothelioma (MPM) cell lines, NCI-H2052 and NCI-H28 displayed a marked increase in phospho-Akt levels after treatment with proteasome inhibitors. Moreover, transfection of the constitutively 
active -Akt into NCI-H2452 cells desensitized NCI-H2452 cells to the apoptosis induced by either TRAIL or MG132 alone or in combination. It was experimentally verified that caspase proteolytically processed Akt protein (Yuan et al., 2013). Protein Kinase C (PKCE) is frequently overexpressed in different cancers and it has been shown that treating cancer cells with proteasome inhibitor induced a partial decline in levels of PKCE. Combinatorially treated glioma cells with TRAIL and proteasome inhibitors induced PKC $\varepsilon$ catalytic fragment accumulation and considerably reduced its mRNA and protein levels. Overexpression of PKCED383A mutant (caspase-resistant) impaired synergistic effects of TRAIL and proteasome inhibitors. AKT and XIAP expression was also reduced in a PKCE-dependent manner in cells treated with TRAIL and proteasome inhibitor (Kahana et al., 2011). Cancerous inhibitor of protein phosphatase $2 \mathrm{~A}$ (CIP2A) is another oncogene frequently overexpressed in different cancers and negatively regulated protein phosphatase-2a (PP2a). PP2a negatively modulated activity of Akt by dephosphorylating it. Combining humanized agonistic antihuman death receptor 5 antibody with bortezomib dramatically inhibited CIP2A that consequently enhanced PP2a mediated inhibitory effects on Akt (Chen et al., 2011).

\section{NFKB}

It has lately been persuasively revealed that CD34(-) acute myeloid leukemia (AML) cells are more bortezomib sensitive as compared to CD34(+) AML cells. Detailed insights indicated that dysregulated NFKB activity in CD34(+) AML cells was the underlying cause. Treating these cells with IKK inhibitor BMS-345541 improved bortezomib induced activity. MCL-1 was also noted be overexpressed and targeted inhibition using siRNA or Obatoclax also helped in improving bortezomib and TRAIL mediated killing effects (Bosman et al., 2013).

\section{Mice Based Studies}

Pegylated liposomal formulation is an efficient carrier of sTRAIL and reported to work with notable synergy in combination with bortezomib in mice xenografted with neuroblastoma cells (Loi et al., 2014).

\section{Conclusion}

Although significant advancements have been made in developing a deeper understanding of the synergistic effects of Bortezomib and TRAIL, however there are still outstanding questions which need detailed investigation. These outstanding questions include how the bioavailability of bortezomib to the target site can be improved, as drug influx, efflux, biodegradation are some of the phenomena which reduce overall availability of bortezomib to the target sites. Another notable divergence while comparing the efficiency of bortezomib in preclinical and clinical trials is the difference in maximal tolerable dosages. Maximal tolerable doses are higher in mice of $1.0 \mathrm{mg} / \mathrm{kg}$ (approximately equal to $3.7 \mathrm{mg} / \mathrm{m}^{2}$ ) as compared to dosages given to human patients of 1.3 $\mathrm{mg} / \mathrm{m}^{2}$ (approximately equal to $0.35 \mathrm{mg} / \mathrm{kg}$ ). Moreover, intratumoral bioavailability needs to be calculated to further identify delivery systems which can effectively carry drug to the site. Surprisingly rapidly emerging resistance against bortezomib is also a major concern as evidenced by overexpression of the $\beta 5$ proteasome subunit unit and mutations in the $\beta 5$ in cancer cells chronically exposed to bortezomib.

\section{References}

Bosman MC, Schuringa JJ, Quax WJ, Vellenga E (2013). Bortezomib sensitivity of acute myeloid leukemia CD34(+) cells can be enhanced by targeting the persisting activity of NF- $x \mathrm{~B}$ and the accumulation of MCL-1. Exp Hematol, 41, 530-8.

Bross PF, Kane R, Farrell AT, et al (2004). Approval summary for bortezomib for injection in the treatment of multiple myeloma. Clin Cancer Res, 10, 3954-64.

Bullenkamp J, Raulf N, Ayaz B, et al (2014). Bortezomib sensitises TRAIL-resistant HPV-positive head and neck cancer cells to TRAIL through a caspase-dependent, E6independent mechanism. Cell Death Dis, 23, 1489.

Bychkov ML, Gasparian ME, Dolgikh DA, Kirpichnikov MP (2014). Combination of TRAIL with bortezomib shifted apoptotic signaling from DR4 to DR5 Death Receptor by selective internalization and degradation of DR4. PLoS One, 9, 109756.

Chauhan D, Catley L, Li G, Podar K, Hideshima T, Velankar M et al. (2005). A novel orally active proteasome inhibitor induces apoptosis in multiple myeloma cells with mechanisms distinct from Bortezomib. Cancer Cell, 8, 407-19.

Chen KF, Yu HC, Liu CY, et al (2011). Bortezomib sensitizes HCC cells to CS-1008, an antihuman death receptor 5 antibody, through the inhibition of CIP2A. Mol Cancer Ther, 10, 892-901.

Gillissen B, Richter A, Richter A, Overkamp T, Essmann F, Hemmati PG, Preissner R, Belka C, Daniel PT (2013). Targeted therapy of the XIAP/proteasome pathway overcomes TRAIL-resistance in carcinoma by switching apoptosis signaling to a Bax/Bak-independent 'type I' mode. Cell Death Dis, 23, 643.

Kahana S, Finniss S, Cazacu S, et al (2011). Proteasome inhibitors sensitize glioma cells and glioma stem cells to TRAILinduced apoptosis by PKCE-dependent downregulation of AKT and XIAP expressions. Cell Signal, 23, 1348-57.

Koschny R, Boehm C, Sprick MR, et al (2014). Bortezomib sensitizes primary meningioma cells to TRAIL-induced apoptosis by enhancing formation of the death-inducing signaling complex. J Neuropathol Exp Neurol, 73, 1034-46.

Laussmann MA, Passante E, Hellwig CT, (2012). Proteasome inhibition can impair caspase-8 activation upon submaximal stimulation of apoptotic tumor necrosis factor-related apoptosis inducing ligand (TRAIL) signaling. J Biol Chem, 287, 14402-11.

Loi M, Becherini P, Emionite L, et al (2014). sTRAIL coupled to liposomes improves its pharmacokinetic profile and overcomes neuroblastoma tumour resistance in combination with Bortezomib. J Control Release, 28, 157-66.

Rapino F, Naumann I, Fulda S (2013). Bortezomib antagonizes microtubule-interfering drug-induced apoptosis by inhibiting G2/M transition and MCL-1 degradation. Cell Death Dis, 21, 925 .

Somasekharan SP, Koc M, Morizot A, et al (2013). TRAIL promotes membrane blebbing, detachment and migration 
Muhammad Zahid Qureshi et al

of cells displaying a dysfunctional intrinsic pathway of apoptosis. Apoptosis, 18, 324-36.

Tuthill MH, Montinaro A, Zinngrebe J, et al (2014). TRAIL-R2specific antibodies and recombinant TRAIL can synergise to kill cancer cells. Oncogene. [Epub ahead of print].

Unterkircher T, Cristofanon S, Vellanki SH, Nonnenmacher L, Karpel-Massler G, Wirtz CR, Debatin KM, Fulda S (2011). Bortezomib primes glioblastoma, including glioblastoma stem cells, for TRAIL by increasing tBid stability and mitochondrial apoptosis. Clin Cancer Res, 17, 4019-30.

Xu L, Su L, Liu X (2012). PKCס regulates death receptor 5 expression induced by PS-341 through ATF4-ATF3/CHOP axis in human lung cancer cells. Mol Cancer Ther, 11, 2174-82.

Yuan BZ, Chapman J, Ding M, et al (2013). TRAIL and proteasome inhibitors combination induces a robust apoptosis in human malignant pleural mesothelioma cells through Mcl-1 and Akt protein cleavages. BMC Cancer, 22, 140 .

Zhao B, Li L, Cui K, et al (2011). Mechanisms of TRAIL and gemcitabine induction of pancreatic cancer cell apoptosis. Asian Pac J Cancer Prev, 12, 2675-8. 